\title{
The Role of BRAF Gene in Cancer: Literature Review and Future Directions
}

\author{
Ricardo Hsieh *
}

Associate Research Scientist, Institute of Tropical Medicine, University of São Paulo, São Paulo, Brazil

\begin{abstract}
The BRAF gene encodes a protein belonging to the RAF family of serine/threonine protein kinases. This protein plays an important role in regulating the MAP kinase signaling pathway, which is involved in cellular development, differentiation, division, proliferation, secretion, inflammatory responses and apoptosis in mammalian cells.

Since 2002, the mutation of valine 600 to glutamic acid (V600E) is the most prevalent, and it is found to be recurrent in many cancer types. It is frequently identified cancer-causing mutation in melanoma, colorectal cancer, thyroid carcinoma, non-small cell lung carcinoma, hairy cell leukemia, non-Hodgkin lymphoma, glioneuronal tumors, hepatocellular carcinoma, adenocarcinoma of lung, ovarian cancer, and also others malignancies and some cancer metastasis.

In the early 1990s, some researchers began studying MAP kinase signaling pathway involved in controlling cell growth and its role in cancer, and it helped identify targets for new classes of cancer therapy. Later BRAF mutation was found in over $50 \%$ of melanomas. The overactive BRAF protein expression looked like an attractive drug target. Elucidating the detailed molecular structure of the mutant protein helped pharmaceutical companies developed selective inhibitors of mutated BRAF, including Vemurafenib and Dabrafenib, which have been approved to treat melanoma by the Food and Drug Administration (FDA).

In addition, there is a growing number of targeted agents that are being evaluated to treat various BRAF-mutant advanced cancer (especially melanoma, lung, thyroid and colorectal cancer), including other RAF kinase inhibitors and/or MEK inhibitors.

The standard therapy of inhibition of BRAF mutation in advanced melanoma and/or others malignancies, improved clinical benefit compared to chemotherapy. In the meantime, intrinsic and acquired resistances are still key challenges by using these drugs. The future research is heading to understand the mechanisms of the resistance, therefore it will help us to understand diseases biology and continuously bringing new therapeutic strategies for melanoma and/or others malignancies, including other drugs combination and next-generation of BRAF inhibitors.
\end{abstract}

Keywords: BRAF, MAPK, V600E, Mutation, Cancer, Therapy.

\section{BRAF GENE: AN OVERVIEW}

Since 1983, RAF family kinases have been associated with some types of cancer etiopathogenesis. Firstly, virus-induced rapidly accelerated fibrosarcoma ( $\mathrm{v}-\mathrm{RAF}$ ) was described and identified as a murine retroviral oncogene with a mammalian cell homologue, named CRAF, also known as RAF1, that has the ability to transform NIHT3T cells. In the meantime, other researchers have discovered v$\mathrm{MIL}$, an avian retroviral oncogene, orthologous to $\mathrm{V}$ RAF. One year later, both V-RAF and V-MIL were first oncoproteins identified to have serine-threonine kinase activity in cell division. Two related kinase genes ARAF and BRAF to CRAF were subsequently found in mice and human cells, and later were found to be commonly mutated in cancer $[1,2]$.

These three RAF proteins are composed of three conserved domains known as conserved regions 1,2 , 3 (CR1, CR2, CR3, respectively) in mammalian cells.

*Address correspondence to this author at the Avenida Doutor Enéas de Carvalho Aguiar, NO. 500, Prédio II, 20 Andar, São Paulo, SP, ZIP CODE: 05403-000, Brazil; Tel: +551130617064; Mob: +5511981932512;

E-mail: ricardoxue@gmail.com, r.hsieh@usp.br
Hence, CR1 has 131aa and it contains cysteine-rich domain and most of RAS GTP-binding self-regulatory domain; CR2 has only 16aa, is a serine/threonine-rich hinge region, and CR3 has 293aa and it is a catalytic serine/threonine protein kinase domain that phosphorylates a consensus sequence on protein substrates in cell cycle. In normal physiology, all three RAF kinases have important roles, however, BRAF is the most predominant RAF kinase that is altered in many different diseases and cancer [3].

The BRAF ( $v$-RAF murine sarcoma viral oncogene homolog B; B-type raf kinase) is located on chromosome $7 q 34$. This gene encodes a cytoplasmatic serine-threonine kinase that mediates the activation of the mitogen-activated protein kinase (MAPK) signaling pathway involved in cellular development, differentiation, division, proliferation, migration, metastasis, secretion, angiogenesis, inflammatory responses and apoptosis in mammalian cells $[2,4,5]$.

\section{BRAF GENE: THE ROLE IN MAPK PATHWAY}

The MAPK pathway is responsible to modulate extracellular signals to control cell growth, proliferation, 
differentiation, migration and apoptosis, through a signaling cascade that is initiated by the binding of growth factors, cytokines or mitogens to their receptors. Ligand-mediated activation of receptor tyrosine kinase results in activation of RAS through RAS GTPase, which recruits and activates some proteins necessary for the propagation of receptor signals, such as BRAF and phosphatidylinositol 3-kinase (PI3K). The BRAF activation happens through a complex process, requiring lipid and protein binding; conformational changes; and regulation of phosphorylate and dephosphorylate events. Once BRAF is phosphorylated, it forms a dimer and acts as a serine threonine-specific protein kinase, however in the inactive form, the BRAF $\mathrm{N}$-terminal regulatory domain autoinhibits the $\mathrm{C}$-terminal kinase domain. Both dimers are further stabilized by 14-3-3 protein heterodimers. The phosphorylated BRAF allows the activation of MEK1/2 (MAP kinase/ERK kinase 1/2) just upstream of ERK 1/2 (extracellular signal-regulated kinases), therefore MEK $1 / 2$ positively regulates the ERK $1 / 2$ by phosphorylation. Lastly, ERK1/2 can directly phosphorylate downstream transcription factor, leading to an increase in the cell function in a variety of ways including transcriptional programs and cell growth and proliferation [3,6-8].

\section{BRAF GENE MUTATION: CANCER AND OTHER DISEASES}

In 2002, a sequencing study performed by the Cancer Genome Project at the Sanger Institute identified a high frequency of BRAF point mutation in melanoma and other human cancers. Thus, this groundbreaking discovery initiated many scientific researches dissecting the role of BRAF and the MAPK pathway in cancer and other diseases pathogenesis $[1,9]$.

The oncogenic activation of the MAPK signaling pathway is very common in human cancers and it occurs by multiple mechanisms, including: mutational activation of RAS-GTPases; point mutation and fusions in BRAF; dysregulation of receptor tyrosine kinases; and also genetic inactivation of RAS-GTPase activating protein neurofibromin-1 (NF-1) [10]. The substitution of a valine for a glutamic acid at position 600 (V600E) is the most common mutation found in BRAF, accounting approximately $80 \%[3,9,11]$.

Although 200 BRAF-mutant alleles have been identified in human cancer, almost 30 different mutation of BRAF gene have been functionally described. Thus, BRAF mutations can be categorized into 3 classes based on their effect on BRAF gene activity: Class 1 BRAF mutations are RAS-independent and behave as active monomer. Class 2 mutant BRAF functions as an active dimer, both Class 1 and 2 mutant BRAF proteins are independent of upstream stimuli for growth and proliferation in malignant tumors. On the other hand, Class 3 mutant BRAF proteins depend directly on RAS signaling for its activation [3].

According to the literature, it is shown that oncogenic BRAF gene fusion can induce activation of BRAF protein in melanomas and other tumors. The fusion consists BRAF kinase domain fused with $\mathrm{N}$ terminal partner genes such as: SOX10; AGK; SEPT3, which result in an alteration of the BRAF copy number and activity, independently of common missense BRAF mutation [3].

Therefore, BRAF gene is one of the most commonly mutated oncogenes in approximately $8 \%$ of all human tumors including melanomas (over 50\%); thyroid cancer (10-70\%); colorectal cancers (over 10-20\%); non-small cell lung cancer (3-6\%); hairy cell leukemia $(100 \%)[4,5,11,12]$. Other human disorders besides cancer are also associated with BRAF and CRAF mutation such as the Noonan and Leopard syndromes, which have developmental defects referred as RASopathies [13].

\subsection{Melanoma}

Melanoma are malignant neoplasm that develop from melanocytes localized in the skin or mucosa, some of them come from pre-existing nevi, and they represent a significant and increasing public health concern and research subject [14-16]. Although melanoma represents $1 \%$ of all diagnosed skin cancer, it is the main cause of most skin cancer-related deaths, with a 5 year survival rate $20 \%$. Cutaneous melanomas are the most common type, followed by mucosal melanomas and lastly the ocular melanoma, although primary mucosal melanomas are rare, they have a worse prognosis compared to the cutaneous counterpart [14,15,17-19].

Many different signaling pathways are altered during the melanoma carcinogenesis, which change normal melanocytes into neoplastic cells, including: MAPK; PI3K; pRb and p53 pathways [20]. It is known that mutated V600E strongly activates MAPK pathway in melanocytes culture, eventually inducing senescence, however it is also found in up to $80 \%$ of human benign moles and nevi, which are considered senescent clones melanocytes. In the melanoma 
formation and progression, it can be accelerated by INK4a and PTEN deficiency and/or concomitant UV exposure [9].

The most common mutation is the substitution of glutamic acid for valine at codon 600 , detected in around $75-90 \%$ of all BRAF mutated-positive melanoma. The second most common mutation is V600K, which is the substitution of lysine for valine, representing $5-8 \%$ followed by V600R, approximately $1 \%$ and $\mathrm{V} 600 \mathrm{M}<1 \%$ [Catalogue of Somatic Mutations in Cancer (COSMIC)- http//:www.sanger.ac.uk/cosmic] $[6,7]$.

According to the literature, single-point mutations in the gene encoding BRAF function occur in $40-60 \%$ of cutaneous melanoma [21]. These same mutations are detected in approximately $50 \%$ of lesions from metastatic melanomas [22]. A mutational profiling of 446 melanomas, BRAF mutation was present in $42 \%$ of cases [23]. A research detecting BRAF and other genes mutations in Russian melanoma patients using LNA PCR clamp and biochip analysis found out BRAF mutation detected by biochip assay in $17,8 \%$ among 253 melanoma specimens. Interestingly, in their results, women showed a higher frequency of this mutation, compared to men [18]. A mutational analysis of 50 tumor samples of Acral lentiginous melanomas observed BRAF mutation in $30 \%$ of specimens [24], although this mutation has been shown to be more common in non-acral melanomas [25]. Additionally, in the mutational status study of BRAF (codon 600) gene in POMM, we observed point mutation in 3 out of 14 cases, and it was screened by pyrosequencing method [26].

In our retrospective studies, we found out BRAF protein expression was positive in $57.14 \%$ of cases Acral Lentiginous Melanoma (ALM) [27] and $74.28 \%$ of cases were positive for BRAF in Primary Oral Mucosal Melanoma (POMM) [17], in both studies we evaluated the protein expression by immunohistochemistry.

\subsection{Gastrointestinal Cancers}

\section{Colorectal Cancer}

Colorectal Cancer (CRC) is the third most common type of malignancy in adults around the world, and it is considered as the second cause of mortality by cancer accounting over 6,000 million of deaths per year $[28,29]$. Although the number of deaths caused by CRC has been decreasing worldwide, it has been shown an increasing of the mortality among young adults $(<50$ years old) [2].
In cancer model studies, the identification of mutational activated KRAS and BRAF alleles in several tumors models explains the importance of MAPK signaling pathway in tumorigenesis. Several reports have demonstrated that MAPK activation through oncogenic RAS and mutated BRAF seems to be involved in promoting cellular invasiveness in different cancer models. In sporadic colorectal cancer KRAS and BRAF mutation have an inversed association, it suggests that each mutation can induce similar cellular effects and signal through the same pathway, thus their mutations do not occur concomitantly, because their combined signaling is incompatible with cell proliferation [6].

The BRAF V600E accounts over $80 \%$ of all reported BRAF mutations in this type of tumor, implicating the constitutive activation of BRAF. Therefore, BRAF mutation is less frequent than KRAS in SCRC, and its mutation is more likely to develop in the right side of colon and is also high associated to metastasis. BRAF is considered a poor prognosis marker in Sporadic Colorectal Cancer (SCRC), because it has a $10 \%$ of frequency that occurs in stage II and III [6], and also associated with worse overall survival [4].

Recently, The American Association for Cancer Research Project Genomics Evi-dence Neoplasia Information Exchange (AACR Project GENIE) involved ten institutions participating in this project, and all institutions performed next-generation sequencing assays for the detection of somatic mutations in cancer. This dataset included 5961 colorectal adenocarcinoma specimens and somatic mutations in RAS-MAPK pathway genes, including: KRAS, NRAS, HRAS, BRAF, MAP2K1, RAF1, and PTPN11 were evaluated. They classified BRAF mutation according to the mechanism of pathway activation: Ras-independent monomers (V600E, class 1); Ras-independent dimers (class 2); Ras-dependent and kinase-impaired activations (class 3 ). Among their findings, a total of 555 pathogenic BRAF mutations were identified in 554 of 5795 specimens $(10 \%)$ : class $1(82 \%)$; class $2(6 \%)$ and class $3(11 \%)$ [30].

\section{Pancreatic Cancer}

Pancreatic Cancer (PC) is considered one of the top leading causes of mortality in some eastern and western countries, with a $10 \%$ of 5 -year survival rate $[31,32]$. PC can be grossly divided into two groups: (1) Exocrine pancreatic neoplasms, which include all tumors related to the pancreatic duct, acinar cells, and their stem cell (Pancreatic ductal adenocarcinoma; 
intraductal papillary mucinous tumor; mucinous cystic tumor; serous cystic tumor), representing $95 \%$ of all PC. (2) Neoplasm of the endocrine pancreas, comprising $5 \%$ of $\mathrm{PC}$, such as islet cell tumors (Pancreatic endocrine tumor; pancreatic acinar cell carcinoma; pancreatoblastoma; solid pseudopapillary tumor) [33]. This poor prognosis neoplasm needs for the development of effective diagnosis and therapeutics to improve patient survival, by understanding of molecular pathobiology and new insights into its pathogenesis [31,33]. Up to $90 \%$ of PC has KRAS mutation and it is characterized by constitutive activation of MAPK pathway, additionally, it may be responsible for pancreatic cells to be transformed to cancer cells $[31,34]$.

A Whole-exome sequencing (WES) study of pancreatic neoplasm with acinar differentiation found alteration in BRAF gene in $13 \%$ of their cases [35]. Later another WES study of pancreatic ductal adenocarcinoma showed BRAF V600E mutations occurring at a frequency of $3 \%$, and it was mutually exclusive with KRAS mutations [36].

A comprehensive genomic profiling analysis of 44 pancreatic acinar cell carcinoma identified rearrangements involving BRAF or RAF1 in $23 \%$ of cases [37]. On the other hand, a molecular analysis of 57 cases of acinar cell carcinoma did not detect any BRAF mutation [38]. Also in a large cohort of 126 pancreatic cancers evaluating the KRAS, BRAF and PIK3CA mutations by pyrosequencing technology no BRAF mutation was found to be existed [32].

\subsection{Thyroid Cancer}

Thyroid Cancer (TC) is considered the most common diagnosed endocrine around the world and accounting for approximately 1 to $2.1 \%$ of all cancers [39-41]. TC can be classified in several histological subtypes: Differentiated thyroid cancers (including papillary and follicular thyroid cancer, both arising from follicular cells), accounting 90 - 95\% of cases; Medullary thyroid cancer (3-5\% of cases), which arises from parafollicular cells; Anaplastic thyroid cancers $(<2 \%$ of cases), it is considered the most aggressive and fatal form can develop from differentiated thyroid cancer that de-differentiates or sometimes it arises de novo $[39,42,43]$.

According to many different researches carried out during past few decades, among the different subtypes of TC, Papillary thyroid cancer (PTC) is the most common neoplasm, accounting $80-95 \%$ of all thyroid cancer $[39,43]$. In the past decades, to understand the molecular signaling involved in the TC tumorigenesis, tumor progression and recurrence, some mutated genes as BRAF, RAS, TERT promoter and TP53 were observed in a significant proportion of cases [39,45-48]. BRAF V600E mutations were the most frequent genetic alterations in TC, representing approximately 20 to $50 \%$ of sporadic PTC $[49,50]$. Due to the limited large cohort evidence, the role of BRAF V600E as a biomarker associated to PTC aggressiveness still remains controversial. Most of studies associated BRAF V600E mutation with poor clinical pathologic outcomes in PTC patients (including: large tumor size; lymph node metastasis; advanced clinical stages; recurrence). On the other hand, other researches did not find any association between BRAF V600E with clinical stage, multicentricity or recurrence $[41,46]$.

Anaplastic thyroid cancer (ATC) is a very rare, aggressive tumor and undifferentiated carcinoma, accounting approximately 1 to $9.8 \%$ or all TC cases $[49,51,52]$. BRAF V600E mutation in observed $13.8 \%$ in ATC cases [52], and AKT1/PIK3CA and EIF1AX comutations with BRAF in $36.36 \%$ in ATC [53].

Finally, detection of BRAF mutation is a useful measure to make reliable diagnosis and treatment strategy, and it has demonstrated a high concordance between $I H C$ and molecular methods for detecting BRAF V600E mutation in FFPE tissues samples [54]. Zhao et al., 2019 compared three different methods and they conclude the COBAS 4800 BRAF V600 test proved to be the most sensitive method $(99.3 \%)$, while the other techniques revealed a sensitivity of $98.6 \%$ for $\mathrm{IHC}$ and $97.2 \%$ for Sanger sequencing [55].

\subsection{Lung Cancers}

Lung Cancer is one of the most common neoplastic disease and a leading cause of cancer mortality worldwide, approximately $18.4 \%$ of all deaths [56-59]. It has been increasing in certain population such as nonsmokers and female in developing country, while declining in males in most developed countries $[56,57]$. The most common subtype is non-small cell lung cancer (NSCLC), accounting about 85\% [58-60]. The majority NSCLC patient are diagnosed at advanced stage, about $50 \%$ of them displaying clinically at stage IV and poor prognosis of a five-year survival rate between less than $20 \%$ [56-60]. The mortality rates still remain high, despite all advances in prevention, diagnosis screening, surgical and medical treatment [56]. 
It is known that acquired genetic alterations in certain driver genes (somatic mutation or chromosomal rearrangements) result in tumour growth and invasiveness, and their discovery represents one of the most important progress in NSCLC [57,59]. BRAF mutation is one of oncogenic driver mutation in NSCLC, which mostly has a low prevalence of only 2 $5 \%$ in caucasian lung cancer [61]. In a retrospective study multicenter study in 65 Chinese patients with NSCLC harboring BRAF mutation from 22 centers, they found 54 patients with BRAF V600E mutation and 11 with non-V600E mutations (K601E; G469S; G469V; G596R; G466R; T599dup) [61]. In another Chinese study, a total of 44 patients with NSCLC were examined for tumor mutation genes in their pathological tissue, and in $9.10 \%$ of cases BRAF mutation was found to be present [60].

In Europe, a Spanish prospective study harbored 224 patients with non-squamous NSCLC, overall $85 \%$ of samples were successfully characterized at DNA and RNA levels. Oncogenic drivers were found in $68 \%$ of them, and $4 \%$ showed BRAF mutation. An Italian prospective study involving 1440 consecutive Sardinian patients diagnosed with lung adenocarcinoma showed $3.2 \%$ of BRAF mutation [56].

In an American huge cohort study, they tested consecutively 8388 patients with advanced NSCLC by performing a plasma-based comprehensive genomic profiling evaluation, and somatic alteration were detected in $86 \%$ of samples, among them, $2.8 \%$ showing BRAF alterations [62]. In the meantime, in a Brazilian study investigated the frequency of somatic mutations in EGFR, KRAS, NRAS, BRAF genes in a cohort of 619 tumors with Lung adenocarcinoma and BRAF mutations were found in $19(3 \%)$ cases [58].

Similarly in Asia, a Vietnamese study performed a parallel sequencing to identify alterations in major drivers genes (EGFR, KRAS, NRAS, BRAF, ALK and ROS) in 350 NSCLC patients, and they observed $2.3 \%$ with BRAF mutation [59]. At the same time in Singapore, they evaluated a total of 174 cases diagnosed with NSCLC and NGS DNA panel was performed in 173 cases, and it was found $2 \%$ with BRAF mutations [63].

In 2019, Dormieux et al. investigated the association between driver oncogene alterations and metastatic patterns on imaging assessment in a large cohort $(\mathrm{N}=550)$ of metastatic lung adenocarcinoma patients in stage IV, among them 47 were BRAF mutated, and in this group, mostly showed pleural and pericardial metastases. Thus, they concluded that the application of correlation between molecular status and metastatic tropism in clinical practice should lead to earlier and more accurate diagnosis and treatment [57].

\subsection{Hairy Cell Leukaemia}

Hairy Cell Leukaemia $(\mathrm{HCL})$ is a very uncommon and indolent chronic B-cell lymphoproliferative malignancy by the presence of distinguishing irregular and long villi around cells, thus, the circulating B-cells with a 'hairy' appearance imparted by surface filopodia and cytoplasmic flaps. It accounts for approximately $2 \%$ of all adult leukaemias and less of $1 \%$ of lymphoid neoplasm [64-67]. Typically, patients with HCL exhibit peripheral cytopenia or pancytopenia, splenomegaly in the absence of significant lymphadenopathy, usually a diffuse infiltration of the spleen, bone marrow and liver by leukemic cells is observed [64-66].

In Australia, they compared the ability of fluorescent single-strand conformations polymorphism (F-SSCP); high resolution melting (HRM) and Sanger sequencing to detect BRAF mutations in 20 cases of $\mathrm{HCL}$, and they found V600E mutation in $94 \% ; 89 \%$ and $72 \%$, respectively. Later, in a Japanese study using a quenching probe method to evaluate 54 patients diagnosed with or suspected of having $\mathrm{HCL}$, they identified BRAF mutation in 18 cases (33.3\%) [66].

More recent, a Spanish study of chronic lymphocytic leukemia patients, putative damage mutations were observed in 25 out of 452 patients (5.5\%), among them, 9 patients $(2 \%)$ showed BRAF mutation.

\section{BRAF INHIBITORS AND CANCER TREATMENT}

According to the literature, KRAS, NRAS and BRAF mutations have been considered among the most important oncogenic drivers in many different neoplasm types, including: melanoma, lung, colorectal and pancreatic cancer, such as LY3009120 a pan-RAF and RAF dimer inhibitor has been shown to inhibit RAS and BRAF mutant cell proliferation in vitro and xenograft tumor growth in vivo [68]. Since 2002, due to the advances in the understanding of BRAF mechanism in many different malignant neoplasms, it was described that mostly its mutation occurs in codon 600 , which replaces a valine to glutamic acid (V600E), thus inhibiting BRAF-V600E would be a great target for these cancers therapy, resulting in many laboratorial and preclinical studies to validate it [69].

In early 2005 , Vemurafenib was first synthetized as a compound with an initial biochemical characterization 
revealing a mild selectivity to BRAF-V600E over the wild-type enzyme [69]. Initial clinical trials with the inhibitor Vemurafenib (PLX4032) treatment in metastatic melanomas showed that patients with tumor carrying the BRAF-V600E mutation resulted in complete or partial tumor regression in the majority of them, improving rates of overall and progression-free survival [70,71] . Although BRAF inhibitors Vemurafenib and Dabrafenib monotherapies have shown efficacy in BRAF-V600E or V600K mutated metastatic melanoma, a study with 704 patients showed that Dabrafenib combined with Trametinib significantly improved overall survival in previously untreated patients compared with Vemurafenib monotherapy, the response rate was $64 \%$ and $51 \%$, respectively [72]. Another study hypothesizing the combined inhibition of BRAF (Vemurafenib) and MEK (Cobimetinib), exhibited results that this combination was associated with a significant improvement in progression-free survival among patients with BRAFV600E mutated metastatic melanoma, however it increased in toxicity [73].

BRAF inhibitor monotherapy seems not to show effectiveness in colorectal cancer (CRC), in a combined BRAF and MEK inhibition study with Dabrafenib and Trametinib in 43 BRAF-V600E mutant CRC patients, it was observed that one patient achieved complete response and $12 \%$ achieved partial response, while $56 \%$ had stable disease as best confirmed response, they concluded that the combination of Dabrafenib and Trametinib has activity in a subset of patient with BRAF-V600E mutant CRC [74]. In a trial by combining BRAF, EGFR and MEK inhibitor in patients with BRAF-V600E mutated CRC, they demonstrated that this combination was tolerable, with promising activity in those patients, however further studies need to optimize strategies inhibiting MAPK pathway to overcome both primary and acquired resistance [75]. Sorafenib is an oral inhibitor of BRAF, VEGFR2 amd PDGFR2-beta, which acts against pancreatic cancer in preclinical models. However, the addition of Sorafenib to concurrent Gemcitabine and radiation therapy showed favorable safety profile in unresectable pancreatic adenocarcinoma [76]. Witkiewicz et al. (2015) used one of pancreatic ductal adenocarcinoma to develop a cell line maintaining the BRAF mutation, and these cells were equally sensitive as MNT1 melanoma cell line to the FDA-approved BRAF inhibitor PLX-4032 [36].

In a preliminary study of metastatic papillary thyroid cancer (PTC) treated with Vemurafenib, among the three evaluated patients, the time to progression was between 11.4 and 13.2 months, thus they conclude that BRAF-V660E mutant kinase is a relevant target for therapy in this patient population [77]. In an open-label non-randomised, phase 2 trial at ten academic centres and hospitals worldwide, analyzing 56 patients with recurrent or metastatic PTC refractory to radioactive iodine and positive BRAF-V600E mutation, Vemurafenib showed antitumor activity [78]. A combination of Dabrafenib and Trametinib treatment in 16 patients with locally advanced or metastatic BRAFV600E mutant Anaplastic Thyroid Cancer, they concluded that this combination is the first regimen demonstrated to have robust clinical activity in this kind of disease and it was well tolerated [79].

In a study of multiple nonmelanoma cancer study, including: 37 NSCLC and 7 anaplastic thyroid cancer treated with Vemurafenib monotherapy, and 27 cases of colorectal cancer treated with Vemurafenib and cetuximab combination therapy, they found that Vemurafenib had preliminary efficacy in NSCLC group [79]. A open-label multicenter phase 2 trial evaluates 57 patients with NSCLC, and it was noticed that Dabrafenib plus Trametinib could represent a new target therapy with robust antitumor activity and manageable safety profile in these patients [80].

In an in vitro study of hairy cell leukemia ( $\mathrm{HCL})$, they concluded that Vemurafenib and Dabrafenib (BRAF inhibitors) exert potent antileukemic activity in $\mathrm{HCL}$ patients; Dabrafenib seems more effective at inducing apoptosis than Vemurafenib; and Dabrafenib is less affected than Vemurafenib by such protective stromal effect and the latter is further reduced by combined BRAF and MEK inhibition [82]. In a multicenter (Italy and U.S.) of Vemurafenib underlying patients with $\mathrm{HCL}$, the overall response rates were $96 \%$ after a median of 8 weeks in Italy group and $100 \%$ after median of 12 weeks in U.S. group, thus they concluded that Vemurafenib was high effective in patients with relapsed or refractory $\mathrm{HCL}$ [83].

\section{FUTURE DIRECTIONS FOR RESEARCH}

The standard therapy of inhibition of BRAF mutated in advanced melanoma and/or others malignancies, improved clinical benefit compared to chemotherapy. Meantime, intrinsic and acquired resistances are still key challenges by using these drugs. The future research is heading to understand the mechanisms of the resistance, therefore it will help us to understand diseases biology and continuously bringing new therapeutic strategies for melanoma and/or others 
malignancies, including other drugs combination and next-generation of BRAF inhibitors.

\section{CONFLICT OF INTEREST}

The author declares there is no conflict of interest.

\section{REFERENCES}

[1] Holderfield M, Deuker MM, McCormick F, McMahon M. Targeting RAF kinases for cancer therapy: BRAF-mutated melanoma and beyond. Nat Rev Cancer [Internet] 2014; 14(7): 455-67. https://doi.org/10.1038/nrc3760

[2] Ducreux M, Chamseddine A, Laurent-Puig P, Smolenschi C, Hollebecque A, Dartigues $P$, et al. Molecular targeted therapy of BRAF -mutant colorectal cancer. Ther Adv Med Oncol [Internet] 2019; 11(7): 175883591985649. https://doi.org/10.1177/1758835919856494

[3] Zaman, Wu, Bivona. Targeting Oncogenic BRAF: Past, Present, and Future. Cancers (Basel) 2019; 11(8): 1197. https://doi.org/10.3390/cancers11081197

[4] Takahashi H, Takahashi M, Ohnuma S, Unno M, Yoshino $\mathrm{Y}$, Ouchi K, et al. microRNA-193a-3p is specifically down-regulated and acts as a tumor suppressor in BRAF-mutated colorectal cancer. BMC Cancer 2017; 17(1): 1-14. https://doi.org/10.1186/s12885-017-3739-x

[5] Chat-Uthai $N$, Vejvisithsakul $P$, Udommethaporn $S$, Meesiri $P$, Danthanawanit C, Wongchai $\mathrm{Y}$, et al. Development of ultra-short PCR assay to reveal BRAF V600 mutation status in Thai colorectal cancer tissues. PLoS One 2018; 13(6): 1-16. https://doi.org/10.1371/journal.pone.0198795

[6] Oikonomou E, Koustas E, Goulielmaki M, Pintzas A. BRAF vs RAS oncogenes: Are mutations of the same pathway equal? Differential signalling and therapeutic implications. Oncotarget 2014; 5(23): 11752-77. https://doi.org/10.18632/oncotarget.2555

[7] Griffin M, Scotto D, Josephs DH, Mele S, Crescioli S, Bax HJ, et al. BRAF inhibitors: resistance and the promise of combination treatments for melanoma braf inhibitors and resistance mechanisms in patients with. Oncotarget 2017; 8(44): 78174-92. https://doi.org/10.18632/oncotarget.19836

[8] Allen A, Qin ACR, Raj N, Wang J, Uddin S, Yao Z, et al. Rare BRAF mutations in pancreatic neuroendocrine tumors may predict response to RAF and MEK inhibition. PLoS One 2019; 14(6): 1-16.

https://doi.org/10.1371/journal.pone.0217399

[9] Arozarena I, Wellbrock C. Overcoming resistance to BRAF inhibitors. Ann Transl Med 2017; 5(19): 1-12. https://doi.org/10.21037/atm.2017.06.09

[10] Wang J, Yao Z, Jonsson P, Allen AN, Qin ACR, Uddin S, et al. A Secondary Mutation in BRAF Confers Resistance to RAF Inhibition in a BRAF V600E -Mutant Brain Tumor. Cancer Discov [Internet] 2018; 8(9): 1130-41. https://doi.org/10.1158/1535-7163.TARG-17-A129

[11] Ko T, Sharma R, Li S. Genome-wide screening identifies novel genes implicated in cellular sensitivity to BRAFV600E expression. Oncogene [Internet] 2019. https://doi.org/10.1038/s41388-019-1022-0

[12] Marranci A, Jiang Z, Vitiello M, Guzzolino E, Comelli L, Sarti S, et al. The landscape of BRAF transcript and protein variants in human cancer. Mol Cancer 2017; 16(1): 5-10. https://doi.org/10.1186/s12943-017-0645-4

[13] Röck R, Mayrhofer JE, Torres-Quesada O, Enzler F, Raffeiner A, Raffeiner $\mathrm{P}$, et al. BRAF inhibitors promote intermediate BRAF(V600E) conformations and binary interactions with activated RAS. Sci Adv 2019; 5(8): eaav8463. https://doi.org/10.1126/sciadv.aav8463

[14] Cheng L, Lopez-Beltran A, Massari F, Maclennan GT, Montironi R. Molecular testing for BRAF mutations to inform melanoma treatment decisions: A move toward precision medicine. Mod Pathol [Internet] 2018; 31(1): 24-38.

https://doi.org/10.1038/modpathol.2017.104
Colombino M, Paliogiannis P, Cossu A, De Re V, Miolo G, Botti $\mathrm{G}$, et al. BRAF Mutations and Dysregulation of the MAP Kinase Pathway Associated to Sinonasal Mucosal Melanomas. J Clin Med 2019; 8(10): 1577. https://doi.org/10.3390/jem8101577

[16] Olbryt M. Molecular background of skin melanoma development and progression: Therapeutic implications. Postep Dermatologii i Alergol 2019; 36(2): 129-38. https://doi.org/10.5114/ada.2019.84590

[17] Hsieh R, Nico MMS, Coutinho-Camillo CM, Buim ME, Sangueza M, Lourenço SV. The CDKN2A and MAP kinase pathways: Molecular roads to primary oral mucosal melanoma. Am J Dermatopathol 2013; 35(2).

https://doi.org/10.1097/DAD.0b013e31825fa1f6

[18] Emelyanova M, Ghukasyan L, Abramov I, Ryabaya $O$ Kudryavtseva A, Sadritdinova A, et al. mutations in Russian melanoma patients using LNA PCR clamp and biochip analysis. Oncotarget 2017; 8(32): 52304-20. https://doi.org/10.18632/oncotarget.17014

[19] Dumaz N, Jouenne F, Delyon J, Mourah S, Bensussan A, Lebbé C. Atypical BRAF and NRAS mutations in mucosal melanoma. Cancers (Basel) 2019; 11(8). https://doi.org/10.3390/cancers11081133

[20] Gutiérrez-Castañeda LD, Nova JA, Tovar-Parra JD. Frequency of mutations in BRAF, NRAS, and KIT in different populations and histological subtypes of melanoma. Melanoma Res 2019; 1. https://doi.org/10.1097/CMR.0000000000000628

[21] Bisschop C, Ter Elst A, Bosman LJ, Platteel I, Jalving M, Van Den Berg A, et al. Rapid BRAF mutation tests in patients with advanced melanoma: Comparison of immunohistochemistry, Droplet Digital PCR, and the Idylla Mutation Platform. Melanoma Res 2018; 28(2): 96-104. https://doi.org/10.1097/CMR.0000000000000421

[22] Louveau B, Delyon J, De Moura CR, Battistella M, Jouenne F, Golmard L, et al. A targeted genomic alteration analysis predicts survival of melanoma patients under BRAF inhibitors. Oncotarget 2019; 10(18): 1669-87. https://doi.org/10.18632/oncotarget.26707

[23] Lokhandwala PM, Tseng LH, Rodriguez E, Zheng G, Pallavajjalla A, Gocke CD, et al. Clinical mutational profiling and categorization of BRAF mutations in melanomas using next generation sequencing. BMC Cancer 2019; 19(1): 1-10. https://doi.org/10.1186/s12885-019-5864-1

[24] Zaremba A, Murali R, Jansen P, Möller I, Sucker A, Paschen A, et al. Clinical and genetic analysis of melanomas arising in acral sites. Eur J Cancer 2019; 119: 66-76.

https://doi.org/10.1016/i.ejca.2019.07.008

[25] Sheen YS, Tan KT, Tse KP, Liao YH, Lin MH, Chen JS, et al. Genetic alterations in primary melanoma in Taiwan. $\mathrm{Br} \mathrm{J}$ Dermatol 2019; 1-9.

[26] Hsieh R, Nico MMS, Camillo CMC, Oliveira KK, Sangueza M, Lourenço SV. Mutational Status of NRAS and BRAF Genes and Protein Expression Analysis in a Series of Primary Oral Mucosal Melanoma. Am J Dermatopathol 2017; 39(2).

https://doi.org/10.1097/DAD.0000000000000605

[27] Fernandes JD, Hsieh R, De Freitas LAR, Brandao MAR, Lourenço S V., Martin Sangueza, et al. MAP kinase pathways: Molecular roads to primary Acral Lentiginous melanoma. Am J Dermatopathol 2015; 37(12): 892-7. https://doi.org/10.1097/DAD.0000000000000317

[28] Yang Y, Wang D, Jin L, Wu G, Bai Z, Wang J, et al. Prognostic value of the combination of microsatellite instability and BRAF mutation in colorectal cancer. Cancer Manag Res 2018; 10: 3911-29. https://doi.org/10.2147/CMAR.S169649

[29] dos Santos W, Sobanski T, de Carvalho AC, Evangelista AF, Matsushita M, Berardinelli GN, et al. Mutation profiling of cancer drivers in Brazilian colorectal cancer. Sci Rep 2019; 9(1): 1-13. https://doi.org/10.1038/s41598-019-49611-1

[30] Costigan DC, Dong F. The extended spectrum of RAS-MAPK pathway mutations in colorectal cancer. Genes Chromosom Cancer 2019; 1-8. https://doi.org/10.1002/gcc.22813 
[31] Furukawa T. Impacts of activation of the mitogen-activated protein kinase pathway in pancreatic cancer. Front Oncol 2015; 5: $1-5$. https://doi.org/10.3389/fonc. 2015.00023

[32] Zhou L, Baba Y, Kitano Y, Miyake K, Zhang X, Yamamura K, et al. KRAS, BRAF, and PIK3CA mutations, and patient prognosis in 126 pancreatic cancers: pyrosequencing technology and literature review. Med Oncol 2016; 33(4): 1-8. https://doi.org/10.1007/s12032-016-0745-9

[33] Matthaios D, Zarogoulidis P, Balgouranidou I, Chatzaki E, Kakolyris S. Molecular pathogenesis of pancreatic cancer and clinical perspectives. Oncology 2011; 81(3-4): 259-72. https://doi.org/10.1159/000334449

[34] Ishimura N, Yamasawa K, Rumi MAK, Kadowaki $Y$, Ishihara S, Amano $\mathrm{Y}$, et al. BRAF and K-ras gene mutations in human pancreatic cancers. Cancer Lett 2003; 199(2): 169-73. https://doi.org/10.1016/S0304-3835(03)00384-7

[35] Jiao Y, Yonescu R, Offerhaus GJA, Klimstra DS, Maitra A, Eshleman JR, et al. Whole-exome sequencing of pancreatic neoplasms with acinar differentiation. J Pathol 2014; 232(4): 42835.

\section{https://doi.org/10.1002/path.4310}

[36] Witkiewicz AK, McMillan EA, Balaji U, Baek GH, Lin WC, Mansour $\mathrm{J}$, et al. Whole-exome sequencing of pancreatic cancer defines genetic diversity and therapeutic targets. Nat Commun [Internet] 2015; 6: 1-11. https://doi.org/10.1038/ncomms 7744

[37] Chmielecki J, Hutchinson KE, Frampton GM, Chalmers ZR, Johnson A, Shi C, et al. Comprehensive genomic profiling of pancreatic acinar cell carcinomas identifies recurrent RAF fusions and frequent inactivation of DNA repair genes. Cancer Discov 2014; 4(12): 1398-405.

https://doi.org/10.1158/2159-8290.CD-14-0617

[38] Bergmann F, Aulmann S, Sipos B, Kloor M, von Heydebreck A, Schweipert J, et al. Acinar cell carcinomas of the pancreas: a molecular analysis in a series of 57 cases. Virchows Arch 2014; 465(6): 661-72. https://doi.org/10.1007/s00428-014-1657-8

[39] Tang K, Lee C. ARTICLE BRAF Mutation in Papillary Thyroid Carcinoma: Pathogenic Role and Clinical Implications. J Chinese Med Assoc [Internet] 2010; 73(3): 113-28. https://doi.org/10.1016/S1726-4901(10)70025-3

[40] Crispo F, Notarangelo T, Pietrafesa M, Lettini G, Landriscina M. BRAF Inhibitors in Thyroid Cancer: Clinical Impact 1-14.

[41] Goh X, Lum J, Peiling S, Chionh SB, Koay E, Chiu L, et al. BRAF mutation in papillary thyroid cancer - Prevalence and clinical correlation in a South - East Asian cohort 2019; 114-23. https://doi.org/10.1111/coa.13238

[42] Rendl G, Rodrigues M, Josef GS, Anton H, Zellinger B, Christian $\mathrm{CH}$. Clinicopathological characteristics of thyroid cancer in the federal state of Salzburg 2017; (540): 540-4.

https://doi.org/10.1007/s00508-017-1207-x

[43] Duan H, Liu X, Ren X, Zhang H, Wu H, Liang Z. Mutation profiles of follicular thyroid tumors by targeted sequencing 2019; 1-10. https://doi.org/10.1186/s13000-019-0817-1

[44] Melo M, Gaspar A, Batista R, Jo M, Costa G, Ribeiro C, et al. TERT, BRAF, and NRAS in Primary Thyroid Cancer and Metastatic Disease 2017; 102: 1898-907. https://doi.org/10.1210/jc.2016-2785

[45] Yan C, Huang M, Li X, Wang T, Ling R. Relationship between BRAF V600E and clinical features in papillary thyroid carcinoma 2019; (5): 988-96. https://doi.org/10.1530/EC-19-0246

[46] Huang M, Yan C, Xiao J, Wang T, Ling R. Relevance and clinicopathologic relationship of BRAF V600E , TERT and NRAS mutations for papillary thyroid carcinoma patients in Northwest China 2019; 6-15.

https://doi.org/10.1186/s13000-019-0849-6

[47] Yu X, Wang J. Key candidate genes associated with BRAF V600E in papillary thyroid carcinoma on microarray analysis 2019; 23369-78.

https://doi.org/10.1002/jcp.28906
[48] Fazeli S, Paal E, Maxwell JH, Burman KD, Nylen ES, Khosla SG. Salutary Response to Targeted Therapy in Anaplastic Thyroid Cancer 2019. https://doi.org/10.1177/2324709619890942

[49] Gao J, Ma XP, Deng FS, Jiang L, Jia WD, Li M. Associations of the BRAF V600E Mutation and PAQR3 Protein Expression with Papillary Thyroid Carcinoma Clinicopathological Features 2019. https://doi.org/10.1007/s12253-019-00779-x

[50] Aghajani MJ, Cooper A, Mcguire H, Jeffries T, Saab J, Ismail K Pembrolizumab for anaplastic thyroid cancer: a case study American Joint Committee on Cancer. Cancer Immunol Immunother [Internet] 2019; (0123456789). https://doi.org/10.1007/s00262-019-02416-7

[51] Bonhomme B, Godbert Y, Perot G, Al Ghuzlan A, Bardet S, Belleannée G, et al. Molecular Pathology of Anaplastic Thyroid Carcinomas: A Retrospective Study of 144 Cases. Thyroid [Internet] 2017; 27(5): 682-92.

\section{https://doi.org/10.1089/thy.2016.0254}

[52] Yoo S, Song YS, Lee EK, Hwang J, Kim HH, Jung G, et al. Integrative analysis of genomic and transcriptomic characteristics associated with progression of aggressive thyroid cancer. Nat Commun [Internet] 2019; 10(1): 2764. https://doi.org/10.1038/s41467-019-10680-5

[53] Zhang $\mathrm{X}$, Wang L, Wang J, Zhao $\mathrm{H}$, Wu J, Liu S, et al Immunohistochemistry is a feasible method to screen BRAF V600E mutation in colorectal and papillary thyroid carcinoma. Exp Mol Pathol [Internet] 2018; 105(1): 153-9. https://doi.org/10.1016/i.yexmp.2018.07.006

[54] Zhao J, Liu P, Yu Y, Zhi J. Comparison of diagnostic methods for the detection of a BRAF mutation in papillary thyroid cancer 2019; 4661-6.

https://doi.org/10.3892/ol.2019.10131

[55] Colombino $M$, Paliogiannis $P$, Cossu A, Santeufemia DA, Sini MC, Casula M, et al. EGFR, KRAS, BRAF, ALK, and CMET genetic alterations in 1440 Sardinian patients with lung adenocarcinoma. BMC Pulm Med [Internet] 2019; 19(1): 209. https://doi.org/10.1186/s12890-019-0964-x

[56] Dormieux A, Mezquita L, Cournede P-H, Lacroix L, Rouleau E, Adam $\mathrm{J}$, et al. Association of metastatic pattern and molecular status in metastatic lung non-small cell lung cancer adenocarcinomas. Ann Oncol [Internet] 2019; 30: vii14. https://doi.org/10.1093/annonc/mdz413.049

[57] Andreis TF, Correa BS, Vianna FS, De-Paris F, Siebert M, Leistner-Segal $\mathrm{S}$, et al. Analysis of Predictive Biomarkers in Patients With Lung Adenocarcinoma From Southern Brazil Reveals a Distinct Profile From Other Regions of the Country. J Glob Oncol [Internet] 2019; 1(5): 1-9. https://doi.org/10.1200/JGO.19.00174

[58] Dang ATH, Tran VU, Tran TT, Thi Pham HA, Le DT, Nguyen L, et al. Actionable Mutation Profiles of Non-Small Cell Lung Cancer patients from Vietnamese population. Sci Rep [Internet] 2020; 10(1): 1-11. https://doi.org/10.1038/s41598-020-59744-3

[59] Song $P$, Yang $D$, Wang $H$, Cui $X$, Si $X$, Zhang $X$, et al. Relationship between the efficacy of immunotherapy and characteristics of specific tumor mutation genes in non-small cell lung cancer patients. Thorac Cancer [Internet] 2020; 17597714.13447.

[60] Mu Y, Yang K, Hao X, Wang Y, Wang L, Liu Y, et al. Clinical Characteristics and Treatment Outcomes of 65 Patients With BRAF-Mutated Non-small Cell Lung Cancer. Front Oncol [Internet] 2020; 10: 1-9. https://doi.org/10.3389/fonc.2020.00603

[61] Mack PC, Banks KC, Espenschied CR. Spectrum of Driver Mutations and Clinical Impact of Circulating Tumor DNA Analysis in Non - Small Cell Lung Cancer: Analysis of Over 8000 Cases 2020; 1 -5.

https://doi.org/10.1002/cncr.32876

[62] Tan AC, Lai GGY, San G, Yu S, Doble B, Hui T, et al. Utility of incorporating next-generation sequencing ( NGS ) in an Asian non- small cell lung cancer ( NSCLC ) population: Incremental yield of actionable alterations and cost-e ff ectiveness analysis. Lung Cancer [Internet] 2020; 139: 207-15. https://doi.org/10.1016/j.lungcan.2019.11.022 
[63] Thomas C, Amanuel B, Finlayson J, Grieu-lacopetta Dvsawne F. BRAF mutation detection in hairy cell leukaemia from archival haematolymphoid specimens. Haematology 2015; 47(4): 349-54. https://doi.org/10.1097/PAT.0000000000000245

[64] Angelova EA, Medeiros LJ, Wang W, Muzzafar T, Lu X, Khoury $\mathrm{JD}$, et al. Clinicopathologic and molecular features in hairy cell leukemia- variant: single institutional experience. Mod Pathol 2018; 31: 1717-32. https://doi.org/10.1038/s41379-018-0093-8

[65] Itamura $H$, Ide $M$, Sato $A$, Sueoka $N$, Eisaburo A, Aya $S$. Identification of the BRAF V600E mutation in Japanese patients with hairy cell leukemia and related diseases using a quenching probe method. Int J Hematol [Internet] 2018; 108: 416-22.

https://doi.org/10.1007/s12185-018-2506-3

[66] Abdel Z, Muwalla F, Jiang L, Foran J. Hairy cell leukemia with CCND1 / IGH fusion gene and BRAF V600E mutation. Leuk Res Reports [Internet] 2020; 13: 100197

https://doi.org/10.1016/j.Irr.2020.100197

[67] Chen SH, Gong X, Zhang Y, Van Horn RD, Yin T, Huber L, et al. RAF inhibitor LY3009120 sensitizes RAS or BRAF mutant cancer to CDK4/6 inhibition by abemaciclib via superior inhibition of phospho-RB and suppression of cyclin D1. Oncogene 2018; 37(6): 821-32.

https://doi.org/10.1038/onc.2017.384

[68] Bollag G, Tsai J, Zhang J, Zhang C, Ibrahim P, Nolop K, et al. Vemurafenib: the first drug approved for BRAF-mutant cancer. Nat Rev Drug Discov [Internet] 2012; 11(11): 873-86. https://doi.org/10.1038/nrd3847

[69] Flaherty KT, Puzanov I, Kim KB, Ribas A, McArthur GA, Sosman $\mathrm{JA}$, et al. Inhibition of Mutated, Activated BRAF in Metastatic Melanoma. N Engl J Med [Internet] 2010; 363(9): 809-19. https://doi.org/10.1056/NEJMoa1002011

[70] Chapman PB, Hauschild A, Robert C, Haanen JB, Ascierto P, Larkin J, et al. Improved Survival with Vemurafenib in Melanoma with BRAF V600E Mutation. N Engl J Med [Internet] 2011; 364(26): 2507-16.

https://doi.org/10.1056/NEJMoa1103782

[71] Robert C, Karaszewska B, Schachter J, Rutkowski P, Mackiewicz A, Stroiakovski D, et al. Improved overall survival in melanoma with combined dabrafenib and trametinib. $\mathrm{N}$ Engl J Med 2015; 372(1): 30-9. https://doi.org/10.1056/NEJMoa1412690

[72] Larkin J, Ascierto PA, Dréno B, Atkinson V, Liszkay G, Maio M, et al. Combined Vemurafenib and Cobimetinib in BRAF -Mutated Melanoma. N Engl J Med [Internet] 2014; 371(20): 1867-76. https://doi.org/10.1056/NEJMoa1408868

[73] Corcoran RB, Atreya CE, Falchook GS, Kwak EL, Ryan DP, Bendell JC, et al. Combined BRAF and MEK Inhibition With Dabrafenib and Trametinib in BRAF V600-Mutant Colorectal Cancer. J Clin Oncol [Internet] 2015; 33(34): 4023-31.

https://doi.org/10.1200/JCO.2015.63.2471
[74] Corcoran RB, André T, Atreya CE, Schellens JHM, Yoshino T, Bendell JC, et al. Combined BRAF, EGFR, and MEK Inhibition in Patients with BRAF V600E -Mutant Colorectal Cancer. Cancer Discov [Internet] 2018; 8(4): 428-43. https://doi.org/10.1158/2159-8290.CD-17-1226

[75] Aparicio J, García-Mora C, Martín M, Petriz ML, Feliu J, Sánchez-Santos ME, et al. A Phase I, Dose-Finding Study of Sorafenib in Combination with Gemcitabine and Radiation Therapy in Patients with Unresectable Pancreatic Adenocarcinoma: A Grupo Español Multidisciplinario en Cáncer Digestivo (GEMCAD) Study. Stemmer SM, editor. PLoS One [Internet] 2014; 9(1): e82209. https://doi.org/10.1371/journal.pone.0082209

[76] Kim KB, Cabanillas ME, Lazar AJ, Williams MD, Sanders DL, llagan JL, et al. Clinical responses to vemurafenib in patients with metastatic papillary thyroid cancer harboring BRAFV600E mutation. Thyroid 2013; 23(10): 1277-83. https://doi.org/10.1089/thy.2013.0057

[77] Brose MS, Cabanillas ME, Cohen EEW, Wirth LJ, Riehl T, Yue $\mathrm{H}$, et al. Vemurafenib in patients with BRAFV600E-positive metastatic or unresectable papillary thyroid cancer refractory to radioactive iodine: a non-randomised, multicentre, open-label, phase 2 trial. Lancet Oncol [Internet] 2016; 17(9): 1272-82. https://doi.org/10.1016/S1470-2045(16)30166-8

[78] Subbiah V, Kreitman RJ, Wainberg ZA, Cho JY, Schellens JHM, Soria JC, et al. Dabrafenib and Trametinib Treatment in Patients With Locally Advanced or Metastatic BRAF V600-Mutant Anaplastic Thyroid Cancer. J Clin Oncol [Internet] 2018; 36(1): 713.

\section{https://doi.org/10.1200/JC0.2017.73.6785}

[79] Hyman DM, Puzanov I, Subbiah V, Faris JE, Chau I, Blay J-Y, et al. Vemurafenib in Multiple Nonmelanoma Cancers with BRAF V600 Mutations. N Engl J Med [Internet] 2015; 373(8): 726-36. https://doi.org/10.1056/NEJMoa1502309

[80] Planchard D, Kim TM, Mazieres J, Quoix E, Riely G, Barlesi F, et al. Dabrafenib in patients with BRAFV600E-positive advanced non-small-cell lung cancer: a single-arm, multicentre, open-label, phase 2 trial. Lancet Oncol [Internet] 2016; 17(5): 642-50. https://doi.org/10.1016/S1470-2045(16)00077-2

[81] Pettirossi V, Santi A, Imperi E, Russo G, Pucciarini A, Bigerna B, et al. BRAF inhibitors reverse the unique molecular signature and phenotype of hairy cell leukemia and exert potent antileukemic activity. Blood [Internet] 2015; 125(8): 1207-16. https://doi.org/10.1182/blood-2014-10-603100

[82] Tiacci E, Park JH, De Carolis L, Chung SS, Broccoli A, Scott S, et al. Targeting Mutant BRAF in Relapsed or Refractory HairyCell Leukemia. N Engl J Med [Internet] 2015; 373(18): 1733-47. https://doi.org/10.1056/NEJMoa1506583

Received on 02-06-2020

Accepted on 08-07-2020

Published on 24-07-2020

DOI: https://doi.org/10.30683/1929-2279.2020.09.03

(C) 2020 Ricardo Hsieh; Licensee Neoplasia Research.

This is an open access article licensed under the terms of the Creative Commons Attribution Non-Commercial License (http://creativecommons.org/licenses/by-nc/3.0/) which permits unrestricted, non-commercial use, distribution and reproduction in any medium, provided the work is properly cited. 